\title{
Regional Lung Mechanics in Pulmonary Disease*
}

\author{
C. J. Martin, $\dagger$ A. C. Young, and KoH Ishikawa \\ (From the Firland Sanatorium and the Department of Physiology and Biophysics, University \\ of Washington, Seattle, Wash.)
}

Recent studies in this laboratory have shown that one lobe of the lung may be used as a tonometer to measure pressure variations within the human thorax (1). These studies demonstrated that negative pressure swings were greater in the intrapleural "space" about the lower lobe than about the upper lobe, and the pressure swings about the upper lobe were in turn greater than those surrounding an esophageal pressure tap. In normal subjects these differences increased with lung volume. Lobar compliance was similar in upper and lower lobes.

In the "diffuse obstructive pulmonary syndrome" (DOPS or clinical emphysema), the overall distensibility of the lungs is increased when measured as a static function (2-4). The so-called "dynamic compliance," however, is rate dependent, as might be expected, and is decreased in the obstructive airway syndrome $(3,4-6)$. The compliance of individual lobes as well as the pressure relationships about them is unknown in this condition. Lungs with pulmonary fibrosis might be expected to have a decreased compliance ( 7 ) but have accentuated pressure differences about the fibrotic portion.

Herein reported are the values for lobar compliance and the distribution of pressures within the thorax in patients having pulmonary disease.

\section{Methods}

The subjects were patients having routine bronchospirometry who volunteered for additional studies. They were classified according to their functional state as out-

* Submitted for publication October 9, 1964; accepted February 11, 1965.

This investigation was supported in part by U. S. Public Health Service research grant no. HE 01892 from the National Heart Institute and in part by a grant from the National Tuberculosis Association-American Thoracic Society.

† Address requests for reprints to Dr. C. J. Martin, Firland Sanatorium, 1704 N. E. 150th St., Seattle 55, Wash. lined in Table I. Normal subjects $(\mathrm{N})$ were less than 40 years of age, without known disease now or in the past in the lung under study. These patients had a maximal breathing capacity (MBC) greater than predicted and a vital capacity greater than $80 \%$ of predicted (8). Their maximal expiratory flow rates (MEF) were greater than $260 \mathrm{~L}$ per minute for the males and $210 \mathrm{~L}$ per minute for the females (9).

A group of older subjects $(O \pm D)$ were mixed in character. Three subjects in this group had mild DOPS with an MEF between 200 and $240 \mathrm{~L}$ per minute and an $\mathrm{MBC}$ ranging from $76 \%$ to $100 \%$ of predicted. None of these subjects had symptoms referable to their obstructive airway disease; none had significant disease in the lung under study. Two had normal flow rates.

All members of the group labeled DOPS (D) had an MEF less than $170 \mathrm{~L}$ per minute, an air velocity index (AVI) less than 0.8 (10), and hyperlucency of the lung fields on $\mathrm{X}$ ray. There was no productive (fibrotic) disease within the lung being studied.

Those cases of obstructive airway disease with localized productive disease in the upper lobe on $X$ ray $(D+F)$ were grouped separately. All members of this group had an MEF less than $160 \mathrm{~L}$ per minute and an AVI less than 0.8. Lung volumes in this group were at the lower border of the normal range.

A group of patients with pulmonary fibrosis (F) had old tuberculosis lesions primarily located in the upper lobe but no obstructive airway disease. One case of bronchiectasis with lower lobe fibrosis was also included. Both flow rates and lung volumes were normal in this group. The site of the productive disease was localized from PA films of the chest.

One patient with severe pleuritis $(P)$ over the diaphragm and lower half of the right chest was included.

All subjects, after insertion of an esophageal and bronchospirometric (Carlens) catheter, were placed in the supine position (1). One lobe was isolated for use as a tonometer by introducing a double lumened radiopaque catheter $(2 \mathrm{~mm}$ o.d.) through one side of the Carlens catheter (Figure 1). One lumen terminated in a balloon allowing rapid blockage of the lobe, whereas the other lumen protruded through the balloon to sample lobar pressures.

With one lobe blocked and acting as a pressure capsule in that hemithorax, the interrupted vital capacity maneuver was performed by each patient. Lobar pressures were measured approximately 0.2 second after cessation of flow at each step. All compliance values were calculated from pressure and volume changes taking place 
TABLE I

Criterion for classification of twenty-five subjects*

\begin{tabular}{|c|c|c|c|c|c|c|}
\hline \multicolumn{2}{|l|}{ Group } & \multirow{2}{*}{$\begin{array}{c}\text { Age } \\
y r s\end{array}$} & \multirow{2}{*}{$\begin{array}{c}\frac{\begin{array}{c}\text { Maximal } \\
\text { expiratory } \\
\text { flow }\end{array}}{L / \min }\end{array}$} & \multirow[t]{2}{*}{$\begin{array}{l}\text { Per cent } \\
\text { predicted } \\
\text { MBC }\end{array}$} & \multirow[t]{2}{*}{$\begin{array}{c}\text { Per cent } \\
\text { predicted } \\
\text { vital } \\
\text { capacity }\end{array}$} & \multirow[t]{2}{*}{$\begin{array}{l}\text { Number } \\
\text { of } \\
\text { subjects }\end{array}$} \\
\hline & & & & & & \\
\hline $\begin{array}{l}\text { Normal } \\
\text { Older with or without mild DOPS } \\
\text { DOPS } \\
\text { DOPS with fibrosis } \\
\text { Pleural disease } \\
\text { Fibrosis }\end{array}$ & $\begin{array}{l}(\mathrm{N}) \\
(\mathrm{O}+\mathrm{D}) \\
(\mathrm{D}) \\
(\mathrm{D}+\mathrm{F}) \\
(\mathrm{P}) \\
(\mathrm{F})\end{array}$ & $\begin{array}{l}31-39 \\
49-60 \\
46-77 \\
38-64 \\
55 \\
50-57\end{array}$ & $\begin{array}{r}>260 \\
>200 \\
<170 \\
<160 \\
140 \\
>260\end{array}$ & $\begin{array}{r}>100 \\
>\quad 76 \\
<85 \\
<85 \\
86 \\
>90\end{array}$ & $\begin{array}{r}>80 \\
>80 \\
>95 \\
>70 \\
83 \\
>100\end{array}$ & $\begin{array}{l}5 \\
5 \\
5 \\
4 \\
1 \\
5\end{array}$ \\
\hline
\end{tabular}

* Abbreviations : MBC = maximal breathing capacity DOPS = diffuse obstructive pulmonary syndrome.

between the functional residual capacity (FRC) and approximately $50 \%$ of the inspiratory capacity. Values for upper lobe compliance were obtained by measuring volume changes in upper lobe and pressure changes within the obstructed lower lobe. Lower lobe compliance values were separately obtained using upper lobe pressures and lower lobe volumes.

Later in each experiment a differential transducer connected between the lobar blocking catheter and the blocked end of the Carlens catheter to the same lung was used to measure differential pressures between upper and lower lobes. Other transducers simultaneously measured pressure differences between a lobe and esophagus or these areas and ambient air.

Volume changes of unblocked lobes and lungs were measured with spirometers fitted with potentiometers. Volumes and pressures were fed into a 4-channel recorder (Sanborn). For the right hemithorax, middle lobe was measured as a part of the lower lobe.

All patients were given Seconal (100 mg), atropine ( $0.06 \mathrm{mg}$ ), and morphine (6 to $10 \mathrm{mg}$ ), and a topical anesthesia ( $5 \%$ cocaine).

\section{Results}

All subjects, except those with localized fibrotic disease, had similar compliance values in lobes having corresponding positions on right and left. The values reported below, therefore, are not separated according to right or left lung. Although two females were used as subjects, their number was small and their findings similar to others with like pathology; thus, no separation was made on the basis of sex in the figures cited below.

Lobar compliance. Compliance of the lung lobes and whole lung in one hemithorax is shown in Table II.

In normal subjects $(\mathrm{N})$ no significant differences were found in compliance between upper and lower lobes.

In the group of older subjects, some with a mild abnormality of expiratory flow rate $(\mathrm{O} \pm \mathrm{D})$, there were no significant differences in compliance between upper and lower lobes $(p>0.05)$, although upper lobe compliance exceeds that for lower. The greatest difference between this group and the younger group $(\mathrm{N})$ was in upper lobe compliance. Indeed, the upper lobe distensibility was significantly greater in this group than in the younger subjects $(p<0.05)$. Lower lobe compliance was not significantly different in the two groups. Total lung compliance calculated from the values for each lobe is $0.093 \pm 0.021 \mathrm{~L}$ and is larger than that for the normal subjects $(p<0.05)$.

In moderate to severe obstructive airway disease (D), static compliance was higher in the upper lobe than in the lower lobe $(p<0.003)$. Upper lobe compliance of this group was also

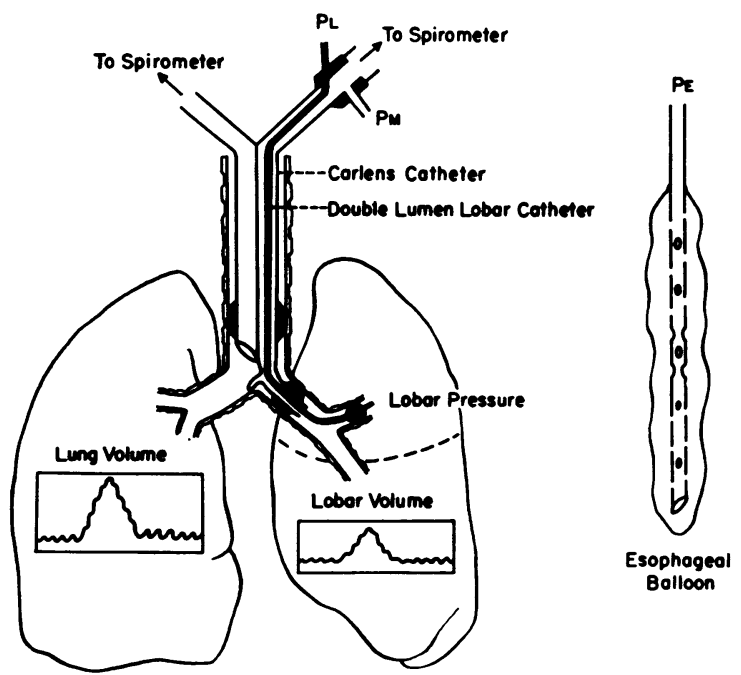

Fig. 1. Diagram of apparatus Showing LObar BLOCKING CATHETER IN PLACE. (Reprinted from J. appl. Physiol. 1964, 19, 823). 
TABLE II

Lobar compliance measured from functional residual capacity to $50 \%$ of inspiratory capacity in the different conditions

\begin{tabular}{|c|c|c|c|c|}
\hline Group & & Upper lobe & Lower lobe & Whole lung \\
\hline $\begin{array}{l}\text { Normal } \\
\text { Older with or without mild DOPS } \\
\text { DOPS } \\
\text { DOPS with fibrosis } \\
\text { Pleural disease } \\
\text { Fibrosis }\end{array}$ & $\begin{array}{l}(\mathrm{N}) \\
(\mathrm{O} \pm \mathrm{D}) \\
(\mathrm{D}) \\
(\mathrm{D}+\mathrm{F}) \\
(\mathrm{P}) \\
(\mathrm{F})\end{array}$ & $\begin{array}{l}L \\
0.037 \pm 0.009 \\
0.050 \pm 0.011 \\
0.062 \pm 0.011 \\
0.036 \pm 0.022 \\
0.034 \\
\text { Lobe involved } \\
0.027 \pm 0.009\end{array}$ & $\begin{array}{l}L \\
0.040 \pm 0.004 \\
0.043 \pm 0.011 \\
0.048 \pm 0.007 \\
0.039 \pm 0.003 \\
0.027 \\
\text { Opposite lobe } \\
0.043 \pm 0.013\end{array}$ & $\begin{array}{l}L \\
0.077 \pm 0.009 \\
0.093 \pm 0.021 \\
0.110 \pm 0.014 \\
0.075 \pm 0.024 \\
0.061 \\
0.070 \pm 0.019\end{array}$ \\
\hline
\end{tabular}

greater than that of the normal group $(\mathrm{p}<0.02)$ and was almost meaningfully larger than that of the $\mathrm{O} \pm \mathrm{D}$ group $(\mathrm{p}<0.06)$. Lower lobe compliance was greater in more severe DOPS than in the normal $(p<0.05)$. Thus, since both lobes have an increased compliance, the lung as a whole is more compliant in DOPS (D) than in normal subjects $(\mathrm{p}<0.05)$.

Four patients in the group $D+F$ with productive disease on $\mathrm{X}$ ray, with lung volumes at the

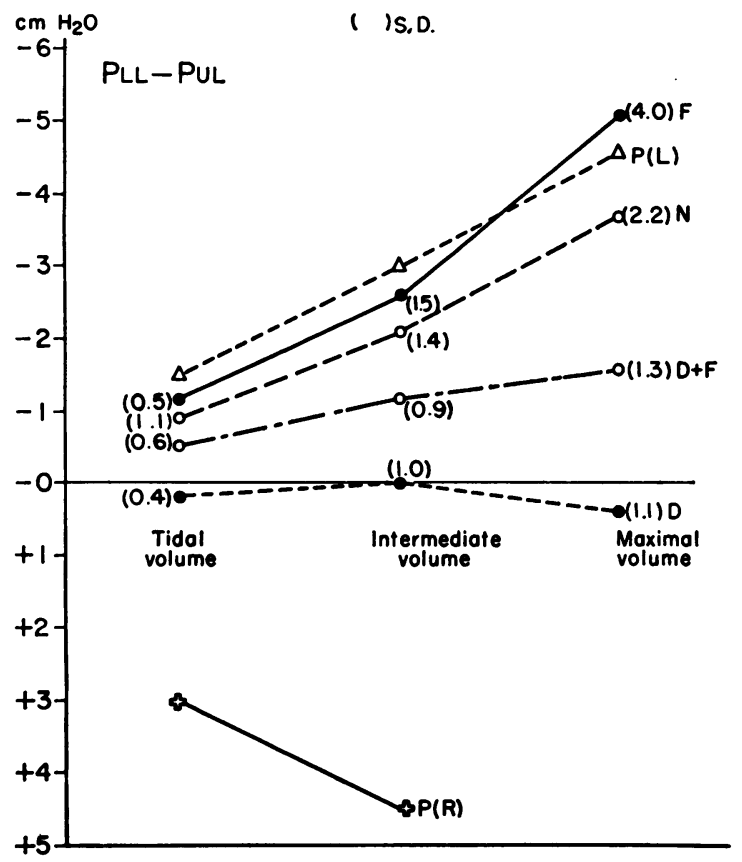

Fig. 2. Pressure Differences betWeen LOWER AND UPPER LOBE (PLL - PUL) IN THE SUBJECTS WITH NORMAL LUNGS (N), PULMONARY FIBROSIS (F), DIFFUSE OBSTRUCTIVE PULMONARY SYNDROME (D), OBSTRUCTIVE PULMONARY SYNDROME AND PULMONARY FIBROSIS $(D+F)$, PLEURAL DISEASE $[P(R)]$, AND A NORMAL LUNG OPPOSITE to oNe With pleural disease $[\mathrm{P}(\mathrm{L})]$. lower border of the normal range, and with obstructive airway disease, had a wide scattering in lobar compliance values. The variation in location and extent of the fibrotic lesion were evidently responsible for the scatter. These subjects have most of their productive disease as a tuberculosis lesion in the upper lobe. The mean value for upper and lower lobe compliance is similar to that in normal younger subjects. But, because of the scatter, this is not significantly different from that seen in the more severe DOPS.

In patients with fibrotic disease $(F)$, the lobe containing most of the fibrosis is listed in the first column of Table II. The mean compliance of the lobe with fibrosis was lower than that in the other lobe on the same side, but individual values were widely scattered, and thus, differences were not significant. The compliance of the fibrotic lobe was significantly less than that in the upper lobe in the $\mathrm{O} \pm \mathrm{D}$ group and in those with DOPS (D) although of similar age. This fibrotic lobe had a significantly lower compliance than the lower lobe in patients with DOPS. Thus, whole lung compliance was lower in fibrosis than in DOPS $(p<$ $0.05)$.

One patient had severe pleural disease encompassing right lower lobe. This lobe had a low compliance (Table II) that could be related to the extent of change observed on $\mathrm{X}$ ray. This lobar distensibility was lower than expected from the functional grouping to which the patient would otherwise have belonged $(\mathrm{O} \pm \mathrm{D})$.

Pressure differences between upper and lower lobes. Pressure differences between upper and lower lobes were measured near functional residual capacity (FRC) at approximately $50 \%$ of the inspiratory capacity and above $70 \%$ of the inspiratory capacity (Figure 2). Volumes were meas- 
ured in the opposite lung while pressure differences were being measured between lobes. The standard deviation of the observations at each point is indicated.

There is progressively larger pressure swing in the lower lobe than upper lobe with increasing lung volume in normal subjects $(N)$, in those with pulmonary fibrosis $(F)$, and in the normal hemithorax of the patient with pleural disease $(\mathrm{P})$. The two groups with obstructive airway disease $(\mathrm{D}+\mathrm{F}$ and $\mathrm{D})$ have little or no differences in pressure between lobes at any volume. A tracing from a subject with obstructive airway disease (Figure 3) shows the difference in pressure between esophagus and room air, and between right lower lobe and right upper lobe, as well as volume changes in the left lung. Little difference in pressure between lobes was observed with volume changes up to $70 \%$ of the inspiratory capacity in the opposite lung.

In the subject with severe pleural disease about right lower lobe, there are smaller pressure swings in this lobe than in the upper lobe on the same side $[P(R)]$, in contrast to the pressure relationships in the normal lung $[\mathrm{P}(\mathrm{L})]$ (Figure 2 ).

Pressure differences between lobe and esophagus. Pressure swings about lower lobes were greater than those in the region of the esophagus at intermediate and large volumes in both the

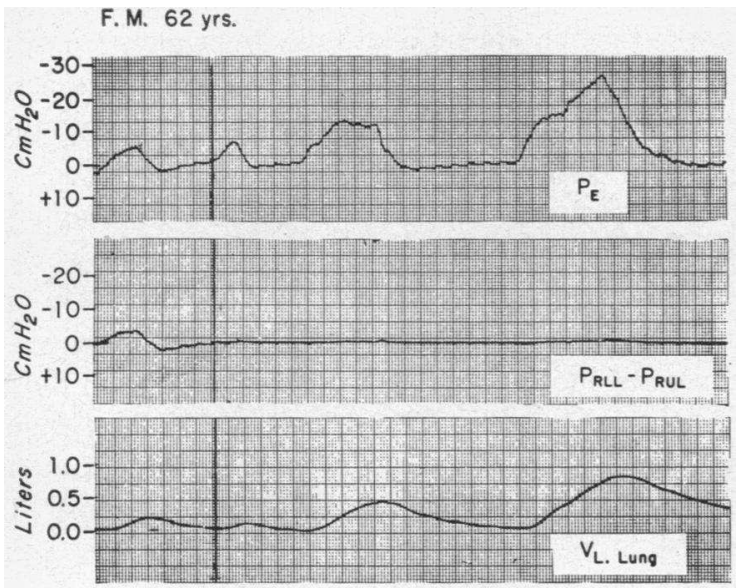

FIG. 3. RECORD SHOWING PRESSURE DIFFERENCES BETWEEN ESOPHAGUS AND ROOM AIR (PE), RIGHT LOWER LOBE AND RIGHT UPPER LOBE $\left(P_{R L L}-P_{R U L}\right)$, AND VOLUME IN LEFT LUNG (Vl. LUNG). Both lobes are blocked and acting as pressure capsules to the right of the heavy vertical line.

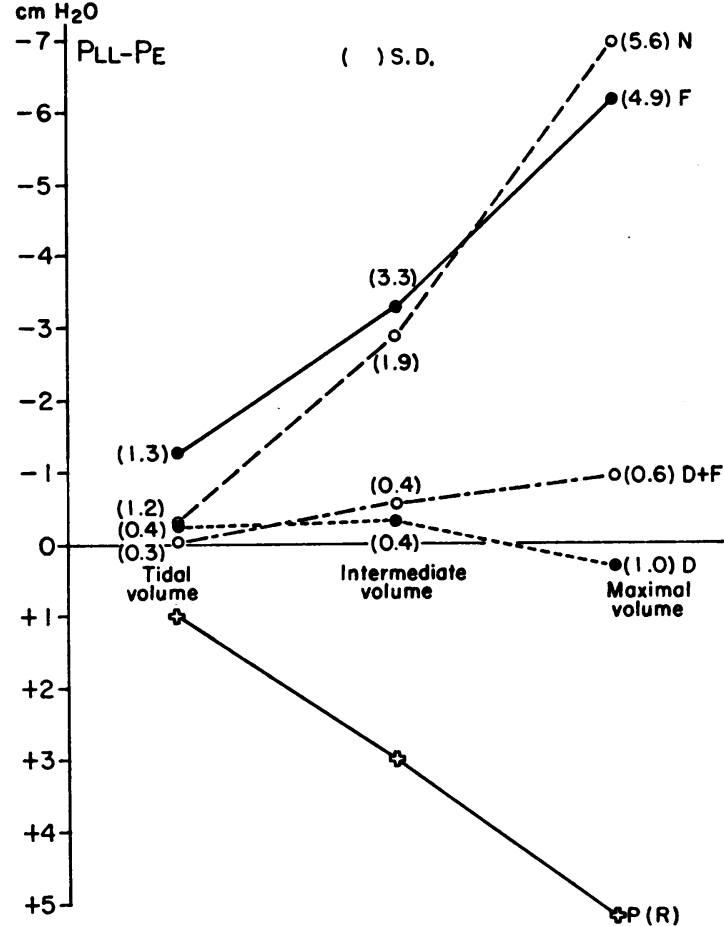

Fig. 4. Pressure differences BetWeen LOWER LOBE AND ESOPHAGUS (PlL - PE) IN THE SUBJeCtS With NORMAL LUNGS (N), PULMONARY FIBROSIS (F), THE DIFFUSE OBSTRUCTIVE PULMONARY SYNDROME (D), OBSTRUCTIVE PULMONARY SY NDROME AND RULMONARY FIBROSIS $(D+F)$, AND PLEURAL DisEASE $[P(R)]$.

normal subjects $(\mathrm{N})$ and in the group with pulmonary fibrosis (F) (Figure 4). Again, in the presence of obstructive airway disease, little difference was found in the magnitude of the pressure swings within the thorax at any lung volume (D, $\mathrm{D}+\mathrm{F})$. With pleural disease about the right lower lobe, the pressure swing in the lobe was much less than that in the esophagus $[\mathrm{P}(\mathrm{R})]$.

The pressure differences between upper lobe and esophagus were similar in direction to those shown between lower lobe and esophagus in Figure 4. In pleural disease pressure swings were greater in esophagus than in the right upper lobe at the larger volume. Obstructive airway disease results in little or no pressure differences between upper lobe and esophagus at any lung volume measured.

The subject with pleural disease demonstrates the remarkable pressure differences that may simultaneously exist within one chest. At the top of Figure 5 is plotted the pressure relationship be- 
tween left lower lobe and right lower lobe. The pressure swing about the left lower lobe greatly exceeded that about the right lower lobe (PLLL PRLL), and these differences increased with lung volume. A normal variation in pressure swing between lobes is present in the left lung (PLLL PluL). The right upper lobe pressure is similar to that in esophagus except at large volumes (PRUL - Pe). Pressure swings in the esophagus were greater than those in the right lower lobe $($ PRLL $-P E)$. Pressure swings in the right upper lobe were greater than in the right lower lobe, and this difference increased with volume (PRLL - Prul).

Pressures were measured simultaneously in right and left lower lobes in four subjects without disease in these regions. Pressure differences between lower lobes were present at large lung volumes. Two subjects had greater pressure swings in the right lower lobe, and two had greater swings in the left.

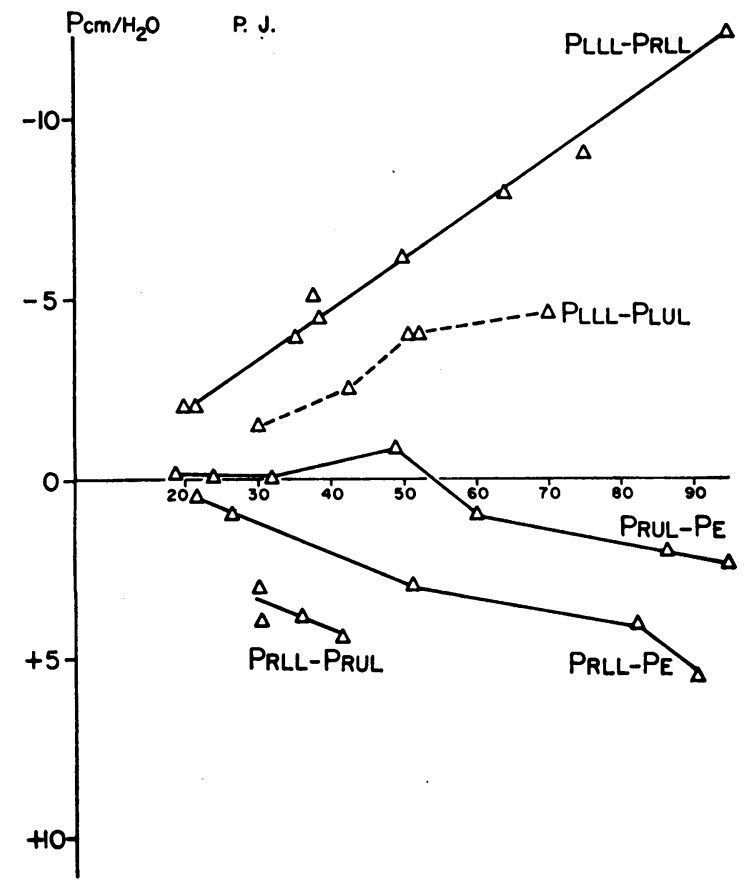

Fig. 5. Pressure differences in the thorax of a PATIENT WITH RIGHT PLEURAL DISEASE BETWEEN LEFT LOWER LOBE AND RIGHT LOWER LOBE $\left(P_{\text {LLL }}-P_{\text {RLL }}\right.$ ), LEFT LOWER LOBE AND LEFT UPPER LOBE $\left(P_{\text {LLL }}-P_{\text {LUL }}\right)$, RIGHT UPPER LOBE AND ESOPHAGUS $\left(P_{\text {RUL }}-P_{E}\right)$, RIGHT LOWER LOBE AND ESOPHAGUS $\left(P_{R L L}-P_{E}\right)$, AND RIGHT LOWER LOBE AND RIGHT UPPER LOBE $\left(P_{\text {RLL }}-P_{\text {RUL }}\right)$.

\section{Discussion}

The lung's elasticity, size, and ability to maintain nonuniform stresses, as well as the ability of chest cage to change shape and volume, determine not only the maximal intrathoracic pressure but also the distribution of pressures within the thorax in a stopped flow system. Lobar volume and ventilation depend upon these relationships, and these in turn determine the alveolar dilution ratio (ADR) or the efficacy of ventilation. Disease states that affect local ventilation changes have been studied by measuring the mechanical properties of lobes of the lung in various pathological states. From these properties inferences may be made as to the changes in ventilation to volume (VA/Vo, ADR) ratios.

This laboratory has shown, with the nitrogen dilution technique, that ventilation to volume ratios are greater in the lower than in the upper lobe in normal subjects (11). An earlier study, as well as the one reported here, has shown that the compliance values for upper and lower lobe are similar in normal subjects, but the pressure swing about the lower lobe is greater than that about the upper lobe (1). This is in keeping with the greater relative ventilation of normal lower lobe and the greater ventilation to volume relationship within that lobe.

In older patients and in those with obstructive disease the higher static lung compliance-most marked in the upper lobe-may be suggestive of a spectrum in which distensibility of the lungs as a whole is progressively increased with age and severity of disease.

With progression of the DOPS and the resultant increased distensibility of the lung, the negative intrathoracic pressure can be maintained only by an increase in lung volume (12). Upper lobe volume must increase more than lower lobe from these compliance figures. The volume of the chest cage, however, is limiting, and eventually the maximal negative pressure that can be developed must diminish (3). Intrathoracic pressure differences might be expected to diminish with the decrease in maximal pressure, but to be as small as noted above in the D group and to be as small in the $\mathrm{D}+\mathrm{F}$ group where the size of the chest cage is more nearly normal implies that the lung is less able to maintain stress gradients. 
Since, with progression of DOPS the increased distensibility of the lung is more marked in the upper lobe, one might expect any architectural change to involve this lobe principally. Most patients dying of obstructive airway disease have a centrilobular or a panlobular emphysema. Centrilobular emphysema is more frequently seen and primarily involves the upper lobe (13). The dissolution of lung tissue seen anatomically in centrilobular emphysema might be expected to have increased distensibility as its physiological counterpart.

Increased static compliance and decreased dynamic compliance have been shown in the lungs of the elderly (14). These changes in the physical properties with age were similar to those seen in DOPS. Bates and Christie have calculated that everyone would show the physiological effects of aging and have obstructive airway disease when 140 years old (15). Whether DOPS has a single anatomical counterpart has been doubted (16), and it can certainly exist without dissolution of lung tissue (17). The distinction between physiological aging and pathology may be diffcult to define.

It is well known that the ADR varies greatly in DOPS, which implies great unevenness of chest wall motion, compliance, airway resistance, or intrathoracic pressure. It appears unlikely that chest wall motion can be a major factor in producing ADR differences since these are presumably mediated by intrathoracic pressure differences. Such pressure differences are not found in patients with DOPS. The difference in ADR between the upper and lower lobes of normal man apparently depends upon unequal pressure relationships within the thorax. In DOPS, the lobar ADR differences depend upon variations in lobar compliance and/or airway resistance. The uniformity of pressure in the chest of those with DOPS means that the esophageal pressure tap provides better mean values for intrathoracic pressure in these subjects than in those who are normal or have pulmonary fibrosis.

We have previously described a model of the lung with fixed, homogeneous airway resistance but with nonlinearities existing either in the intrathoracic pressures or in the mechanical properties of lung units. These nonlinearities would cause a variation in the emptying of different units depending upon the expiratory flow rate (18). Predicted from the model was a decreased nitrogen rise when expiratory flow rates were held constant in contrast to the varying expiratory flow in the normal expiration. This model fitted the results observed in patients with DOPS.

The persistence of unequal emptying at low flow rates in those with DOPS who have uniform intrathoracic pressures must result from the nonuniform, nonlinear mechanical properties of the lung. Whether the major differences in properties are at an inter- or intralobal level is not known at present.

Greater distensibility of the upper lobe and homogeneous intrathoracic pressures in subjects with DOPS must mean that the greater portion of each breath goes into the upper lobe. We have no data on the relative volume of the lobes in this disease nor have we measures of the lobar ventilation to volume ( $\mathrm{VA} / \mathrm{Vo})$ ratios. If the higher $\mathrm{VA} /$ Vo ratio of lower lobe present in normal subjects persists in DOPS, the relative volume of upper to lower lobe would have to increase by $30 \%$.

From the compliance values in patients with pulmonary fibrosis $(F)$, it is likely that more fibrosis was present in the lower lobe than was apparent from $X$ ray. From these values, distribution of lobar ventilation in these subjects with pulmonary fibrosis must resemble that in normal subjects. Lobar compliance in these subjects is comparable to that in normal subjects less than 40 years of age. Since the youngest in this group was 50 , this must represent a fall in compliance from the expected value for this age group. Localized lobar fibrosis canceled the effect of age upon lobar compliance.

In those subjects with pulmonary fibrosis and obstructive disease $(D+F)$ the intralobar fibrosis countered the effect of the obstructive airway disease on compliance. Compliance values were similar to those in young, normal subjects. Pressure swings, however, were more homogeneous throughout the thorax. Homogeneous pressure may be a fortuitous circumstance of the location of the fibrosis; however, it is more likely to result from the properties of the opposite lung (not measured in this group), i.e., a highly compliant lung in one hemithorax may cause homogeneous pressures throughout the chest. Where the lung without 
productive disease has been measured in the presence of DOPS (D), high compliance and homogeneous pressures have been demonstrated. In this group as well as in the group with pulmonary fibrosis in the lung under study $(D+F)$, stress gradients cannot be maintained. A single highly compliant unit in the chest with inability of the lung to maintain stress gradients could lead to uniformly low intrathoracic pressure and be the cause of the diffuse airway obstruction.

The case of pleural disease demonstrates the marked variation in the thoracic pressures that may occur in disease states (Figure 4). Normal pressure relationships were found in the normal left hemithorax. Left lower lobe pressure swings were greater than upper lobe pressure swings, which in turn were greater than those in the esophagus. The left lower lobe pressure swing was significantly greater than that in the right lower lobe. In the region of maximal pleural disease (RLL) the pressure swings were the least marked of anywhere in the thorax.

It is apparent from these results that the use of a single pressure to measure compliance, as a reference for right heart pressure, or as a monitor to describe pressure events within the thorax should be questioned. Furthermore, it is evident that isolated lungs having uniform surrounding pressure will not have the same volume or ventilation relationship as lungs within a normal chest cage. Isolated lungs with DOPS may be the exception to this statement.

Previous work has shown that pressure differences about upper and lower lobes exist in normal man (1). The present study confirms this finding as well as the increase in such differences with an increase in volume. Pressure differences between lower lobes also exist. In pleural disease, differences exist between all points measured. Certainly lungs with such relationships cannot be treated as a hydrostatic system, but rather resemble a viscoelastic solid able to maintain stress differences for limited periods of time. The normal lung has shape that must not be changed if the properties of the unit are to be described by a pressure volume diagram.

\section{Summary}

The compliance of the upper and lower lobe as well as the differences in intrathoracic pressure have been measured.
In the normal lung of man there is no significant difference between the upper and the lower lobe in static compliance. Greater pressure swings occur about the lower lobe than about the upper lobe, which in turn has greater pressure swings than those about an esophageal pressure tap. These pressure differences increase with lung volume. In patients who are older, with and without mild obstructive airway disease, the whole lung compliance, and particularly upper lobe compliance, is greater than in normal subjects. $\mathrm{Pa}$ tients with moderate to severe obstructive airway disease have more compliant lungs than normal subjects, and upper lobe compliance is significantly greater than lower lobe compliance. Homogeneous intrathoracic pressures are found in those patients with obstructive disease at all lung volumes. Patients with pulmonary fibrosis, although older, had a lobar compliance similar to that in younger normal subjects, and intrathoracic pressure differences were similar to those in the normal patients. In the presence of obstructive airway disease the lung or lobe with pulmonary fibrosis may have normal compliance, but it has homogeneous intrathoracic pressures. In pleural disease, differences exist in intrathoracic pressure between all points measured in the chest.

\section{Acknowledgment}

We gratefully acknowledge the technical assistance of Miss Florence Best.

\section{References}

1. Ishikawa, K., C. J. Martin, and A. C. Young. Pressure-volume studies on lung lobes in man. J. appl. Physiol. 1964, 19, 823.

2. Christie, R. V. Elastic properties of the emphysematous lung and their clinical significance. J. clin. Invest. 1934, 13, 295.

3. Mead, J., I. Lindgren, and E. A. Gaensler. The mechanical properties of the lungs in emphysema. J. clin. Invest. 1955, 34, 1005.

4. Cherniack, R. M. The physical properties of the lung in chronic obstructive pulmonary emphysema. J. clin. Invest. 1956, 35, 394.

5. Otis, A. B., and C. McKerrow. Possible mechanism contributing to uneven pulmonary ventilation. Fed. Proc. 1954, 13, 107.

6. Attinger, E. O., M. M. Goldstein, and M. S. Segal. Ventilation in chronic pulmonary emphysema; II. Correlation of compliance and mechanical resistance with routine pulmonary function tests. Amer. Rev. Tuberc. 1956, 74, 220. 
7. Cherniack, R. M. Respiration in Health and Disease. Philadelphia, W. B. Saunders, 1961, p. 18.

8. Baldwin, E. deF., A. Cournand, and D. W. Richards, Jr. Pulmonary insufficiency. I. Physiological classification, clinical methods of analysis, standard values in normal subjects. Medicine (Baltimore) 1948, 27, 243.

9. Goldsmith, J. R. A simple test of maximal expiratory flow for detecting ventilatory obstruction. Amer. Rev. Tuberc. 1958, 78, 180.

10. Gaensler, E. A. Air velocity index: a numerical expression of the functionally effective portion of ventilation. Amer. Rev. Tuberc. 1950, 62, 17.

11. Martin, C. J., and A. C. Young. Lobar ventilation in man. Amer. Rev. Tuberc. 1956, 73, 330.

12. Stead, W. W., D. L. Fry, and R. V. Ebert. The elastic properties of the lung in normal men and in patients with chronic pulmonary emphysema. J. Lab. clin. Med. 1952, 40, 674.
13. Snider, G. L., J. S. Brody, and L. Doctor. Subclinical pulmonary emphysema. Incidence and anatomic patterns. Amer. Rev. resp. Dis. 1962, 85, 666.

14. Frank, N. R., J. Mead, and B. G. Ferris, Jr. The mechanical behavior of the lungs in healthy elderly persons. J. clin. Invest. 1957, 36, 1680.

15. Bates, D. V., and R. V. Christie. Effects of aging upon respiratory function in man in Ciba Foundation Colloquia on Ageing, General Aspects. Boston, Little, Brown, 1955, vol. 1, p. 58.

16. Ting, E. Y., and M. H. Williams, Jr. Mechanics of breathing in chronic obstructive pulmonary disease. Amer. Rev. resp. Dis. 1963, 88, 791.

17. Cohn, J. E., T. H. Cochran, and C. T. Pinney. A comparison of pulmonary physiologic studies with Gough sections of postmortem lungs. Clin. Res. 1959, 7, 128.

18. Young, A. C., C. J. Martin, and W. R. Pace, Jr. Effect of expiratory flow patterns on lung emptying. J. appl. Physiol. 1963, 18, 47. 